establish the unperturbed dimensions of the polymer chain, which depend on bond-lengths and -angles and on hindrance to free rotation. The theory, developed by Flory, enables, furthermore, an estimation to be made of thermodynamic parameters related to the heat and entropy of dilution of the polymer in a given solvent. It is also possible to explain the marked alteration of the viscosity of polyelectrolyte solutions brought about by changes of $p \mathrm{H}$ or ionic strength

Prof. C. E. H. Bawn discussed intrinsic viscosity relationships for polyisobutylene and polystyrene in mixed solvents, some of the intrinsic viscosities being determined over a range of temperatures. These results were used to examine existing theories of the viscosity of polymer solutions and, in particular, the relationships dealt with by Prof. Flory.

Prof. J. A. V. Butler gave an account of viscosity measurements of two polyelectrolyte solutions, these experiments being done at low concentrations and rates of shear. Some of the results were interpreted with the help of an electrostatic theory of charged thread-like particles. Dr. B. A. Toms described a rheological investigation of about 3 per cent solutions of polymethylmethacrylate. The experiments were carried out with the help of a coaxial cylinder elastoviscometer. The rheological behaviour of these solutions can be specified by a viscosity coefficient, a relaxation time and a retardation time. The variability of these parameters indicates that relaxation of shear and of rate of strain are effected by different mechanisms.

\section{THE COUNCIL FOR THE PROMOTION OF FIELD STUDIES}

$\mathrm{A}^{\mathrm{Y}}$

FTER a stringent period of economy caused by financial difficulties, the chairman of the Council for the Promotion of Field Studies, Prof. S. W. Wooldridge, reports that the increased bookings at the field centres and the careful husbanding and disposal of the Council's resources have led to a happier situation and a development of the Council's activities : a grant for equipment has been made to each of the four centres, while the teaching staff of three of them has been augmented by the appointment of a field assistant. In addition, substantial capital expenditure to improve comfort and acommodation has been approved at Dale Fort and Juniper Hall. The fourth annual report of the Council also describes the work at the field centres in $1954^{*}$.

At Dale Fort, marine biology again proved the greatest attraction at the centre, only geography with all its variations providing comparable numbers.

At Skokholm, work has been begun on the annual marking of puffin chicks, while many puffins were ringed to throw light on the movements, attainment of maturity, mortality and expectation of life of these birds.

Students again contributed to the long-term research projects at Flatford Mill; these included investigations into the distribution and feeding habits of flounders at the head of the estuary, and

* Council for the Promotion of Field Studies. Annual Report 1953-54. Pp. $60+8$ plates. (London: Council for the Promotion of Field Studies, 1955.) the study of the vegetational succession taking place on mud-banks forming at the lower edge of Sherbourne Brook.

At Juniper Hall, geographical subjects of all kinds were the main interest of 53 per cent of the total number of students, while biological studies of one kind or another accounted for 39 per cent of the attendance; courses for sixth-form students from grammar schools and public schools formed the most stable element in the centre's economy.

An interesting development at Malham Tarn was the arrangement of a special course for secondary modern school teachers ; this was done in conjunction with the West Riding Local Education Authority.

The report also contains details of some of the specialized work that was carried out at different centres.

\section{NEBULF AND STAR CLUSTERS}

$A$ $T$ the meeting of the Royal Astronomical Society on February 12, 1954, at Burlington House, London, the presidential address (read by Prof. $H$. Dingle in the unavoidable absence of the president, Dr. J. Jackson) was delivered on the occasion of the award by the Society of its Gold Medal to Dr. Walter Baade, of the Mt. Wilson and Palomar Observatories, for his observational work on galactic and extragalactic objects. The address, which has been published in full (Mon. Not. Roy. Astro. Soc., 114, 3 (1954); and also The Observatory, 74, No. 879 (April 1954)), commenced with a brief outline of Baade's work with the reflector of $1 \mathrm{~m}$. aperture at Hamburg Observatory from 1919 until 1931, when he joined the staff of the Mt. Wilson Observatory to work with Hubble on the branch which was his chief interest at Hamburg - the direct photography and photometry of nebulæ and star clusters. Soon after his arrival at Mt. Wilson Observatory he undertook an investigation for determining the distance of the wispy cloud in Cygnus, a region to which he had given special attention when he was at Hamburg and in which he had found that many of the variable stars were eclipsing variables. Three classes of stars were used from Mt. Wilson observations for determining the distance - the eclipsing variables; long-period variables ; and early $B$-type stars-and they indicated distance moduli of $12 \cdot 5,13 \cdot 0$ and $12 \cdot 7$, respectively, the latter being adopted. The question of the absorption of light in space then arose, and from the colour indices of eighty stars of type $B 8$ to $A .5$ a colour excess of 0.25 was found. On the assumption that this was due to pure Rayleigh scattering, Basde deduced that the Cygnus cloud was 2,630 parsecs distant; if no correction for absorption had been applied, a distance of 3,500 parsecs would follow from the adopted modulus. The few Cepheid variables found in the cloud gave very unsatisfactory results : four of long period were so faint that they could not be fitted in with the adopted modulus; two could be explained by heavy absorption; and two others might be much farther off than the cloud. In addition, five short-period cluster-type variables could not be fitted into the general result, but it was possible to explain them as belonging to the general galactic field of such objects.

The address next turned to another branch of Baade's work-that connected with novæ and supernovæ. The discovery by Ritchey in 1917 of a nova 